\title{
Barriers and Enablers to Intervention Uptake and Health Reporting in a Water Intervention Trial in Rural India: A Qualitative Explanatory Study
}

\author{
Sarah L. McGuinness, ${ }^{1 \star}$ Joanne O'Toole, ${ }^{1}$ Darshini Ayton, ${ }^{1}$ Asha Giriyan, ${ }^{2}$ Chetan A. Gaonkar, ${ }^{2}$ Ramkrishna Vhaval, ${ }^{2}$ \\ Allen C. Cheng, ${ }^{1}$ and Karin Leder ${ }^{1}$ \\ ${ }^{1}$ School of Public Health and Preventive Medicine, Monash University, Melbourne, Australia; ${ }^{2}$ The Energy and Resources Institute (TERI), \\ Panaji, India
}

\begin{abstract}
Consumption of unsafe drinking water contributes to the global disease burden, necessitating identification and implementation of effective, acceptable, and sustainable water interventions in resource-limited settings. In a quantitative stepped-wedge cluster randomized trial of a community-based water intervention in rural India, we identified low rates of intervention uptake and reported diarrhea. To better understand and explain these findings, we performed a qualitative study examining barriers and enablers to intervention uptake and health reporting using the COM-B model, where capabilities, opportunities, and motivators interact to generate behavior. We conducted 20 focus groups and one semi-structured interview with participants and four focus groups with data collectors. Multifactorial barriers to intervention uptake included distorted perceptions of water-related health effects, implementation issues that reduced treated water availability; convenience of, and preference for, alternative drinking water sources; delivery of water to plastic storage tanks (perceived as affecting water quality and taste); and resistance to change. Enablers included knowledge of water-related health risks, proximity to tanks, and social opportunity. Barriers to health reporting included variability in interpretation of illness, suspicion regarding the consequences of reporting disease, weariness with repeated questions, and perceived inaction on health data already provided; low survey implementation fidelity was also important. Enablers included surveyor initiatives to encourage reporting and a sense of social responsibility. This qualitative explanatory study allowed better understanding of our quantitative results. It also identified obstacles and facilitators to implementing and evaluating community water interventions, providing insight on how to achieve better intervention uptake and health reporting in future studies.
\end{abstract}

\section{INTRODUCTION}

Unsafe drinking water is a determinant of poor health, ${ }^{1}$ and issues with water availability, affordability, reliability, and contamination contribute to the global burden of disease. ${ }^{2-4}$ In 2012, an estimated 502,000 diarrheal deaths were attributed to insufficient and unsafe drinking water, ${ }^{4}$ and diarrhea remains a leading cause of childhood deaths globally, particularly in low- and middle-income countries (LMICs) such as India. ${ }^{4,5}$ Previous estimates of global access to safe water sources may have been overestimated, ${ }^{6,7}$ with current estimates suggesting that 2.1 billion people worldwide lack access to safely managed drinking water. ${ }^{8}$

Achieving global improvements in access to safe drinking water requires implementation of water quality interventions that are effective, acceptable, and sustainable among target populations. ${ }^{9}$ Good intervention coverage, high uptake, and high adherence (correct, consistent, and sustained use) are essential to realizing health gains from water interventions, as ongoing or intermittent consumption of untreated water potentially undermines any protective effects of water treatment. ${ }^{10-12}$ However, incomplete adherence is often reported in trial settings, intervention use frequently drops once intensive trial promotion and monitoring activities cease, and poor uptake or unsustained use are commonly observed following attempts to bring interventions up to scale. ${ }^{13-16}$

Within the water, sanitation, and hygiene literature, an increasing number of high-quality controlled experimental trials have had disappointing health outcomes. ${ }^{17-21}$ There is growing awareness of the importance of embedding process

* Address correspondence to Sarah L. McGuinness, School of Public Health and Preventive Medicine, Monash University, 553 St. Kilda Rd., Melbourne 3004, Australia. E-mail: sarah.mcguinness@monash.edu evaluation methods into such trials, to allow determination of whether negative results are due to poor intervention efficacy, poor intervention uptake, or failure to implement the intervention as designed. ${ }^{22,23}$ Furthermore, it is recognized that evaluations based solely on quantitative methods provide limited insight into psychosocial, contextual, and technological dimensions that shape and influence target users' perceptions and behavior. ${ }^{10,24}$ The collection of qualitative data from study participants and stakeholders, and integration of these data using a mixed-methods approach, could help to provide a more complete understanding of obstacles or facilitators to intervention uptake and health reporting in water intervention studies. Such qualitative insights could also aid the development of theory-driven behavior change interventions that address these factors head on, inform the adaptation of interventions to different contexts, and allow the nuances of study findings to be better understood. ${ }^{25,26}$

In 2016-2018, we performed a stepped-wedge cluster randomized trial assessing the health effects of improved water quality and access versus improved access alone at a community level in rural villages located in the Athani Taluka of northern Karnataka, India. ${ }^{27}$ Preliminary quantitative analyses indicated a low rate of intervention uptake and a lower than expected prevalence of reported diarrhea. Substantial differences in illness reporting were observed between clusters (villages), with a marked decline in diarrhea reporting over time in three villages compared with relatively stable rates of diarrhea reporting in the remaining village. We used these findings to inform the design and analysis of a qualitative study to help explain our quantitative findings and to inform future studies. Our primary objectives were to examine barriers and enablers to intervention uptake and health reporting. We used the COM-B framework, ${ }^{28}$ a conceptual framework widely adopted in implementation research which sees human 
behavior (B) as the interaction between physical and psychological capabilities (C), social and environmental opportunities $(\mathrm{O})$, and motivators $(\mathrm{M})$.

\section{METHODS}

Study setting. This qualitative study was part of a mixedmethods sequential explanatory study (Figure 1), where a quantitative stepped-wedge cluster randomized interventional trial and a qualitative data component were conducted in two consecutive stages. ${ }^{29}$ The methodology and findings of the stepped wedge trial have previously been described. ${ }^{27,30}$ Briefly, we selected four villages, each with a total population of 2,000-3,000 residents using untreated river water as a primary drinking water source, and enrolled all consenting households. At study commencement, we delivered piped untreated river water (control condition) to newly installed water storage tanks in all villages simultaneously. We then sequentially delivered riverbank filtration-treated water (intervention condition) to tanks in each village in a random order at 12-week intervals. Storage tanks ("project tanks") were 2,000 or $3,000 \mathrm{~L}$ capacity plastic tanks (Sintex Industries), each designed to supply 25-30 households. Although blinding was not possible, we did not overtly tell participants ("householders") when the switch from control to intervention occurred. Data collectors ("surveyors") conducted a baseline health survey (timepoint zero, T0) and delivered hygiene and safe water storage education before the commencement of the study. They also collected health outcome data through five household-level surveys (T1-T5) at 12-week intervals, and reinforced educational messages at each timepoint. Surveys were conducted by surveyor pairs, with three pairs assigned to each village (24 surveyors in total) for the duration of the trial. We deliberately recruited surveyors from the study district; all were fluent in the local language and had a bachelor's degree (men) or a minimum of 10 years of formal schooling (women).

We used findings from a preliminary analysis of T1-T4 survey data to inform the design and analysis of the qualitative explanatory study. This study aimed to provide insight into 1) the low observed rate of intervention uptake in the steppedwedge trial through a qualitative exploration of the drivers of household water choice and the barriers and enablers to the uptake of tank water and 2) the low observed diarrhea prevalence and differences in village reporting trends through a qualitative exploration of barriers and enablers to health reporting. The study had two components-focus groups or semi-structured interviews with selected households and focus groups with surveyors-and was conducted in January 2018, immediately after the quantitative trial. We report our qualitative explanatory study in line with the consolidated criteria for reporting of qualitative research (COREQ) ${ }^{31}$ and include the COREQ checklist as Supplemental Appendix 1.

Methodological orientation. The COM-B model is a conceptual framework developed by Michie and others ${ }^{28}$ following an evaluation and synthesis of 19 existing behavior change frameworks. At the center of the framework is a "behavior system" involving three essential components-capability $(\mathrm{C})$, opportunity $(\mathrm{O})$, and motivation $(\mathrm{M})$-that interact to generate behavior. The COM-B model allows identification of physical and psychological factors (capability), environmental and social contexts (opportunity), and reflective and autonomic processes (motivation) that may act as barriers or enablers to a specific behavioral target. ${ }^{28}$

Data collection. Surveyor focus groups. All surveyors involved in data collection were invited to participate in one of four focus groups according to their allocated village. Surveyors were sent a letter of invitation and a written explanatory statement. Participation was voluntary, with attendance taken as consent to participate. A question guide containing openended questions was developed and piloted with in-country research staff. Questions were designed to explore surveyors' perceptions and beliefs about participants' engagement and health reporting, and the ways in which they had approached participants and solicited answers during study surveys. Questions were mapped to the domains of the COM-B framework (Tables 1 and 2). Because householders were asked during quantitative surveys "Has anyone in the household suffered illness/sickness of any kind in the past week?", with subsequent questions about specific symptoms

\section{Stage 1: Pre-trial activities (Jan 2015 - Sep 2016)}

- Ethics approval, hydrogeological surveys, identification of study sites, stakeholder engagement

- Consultation with community leaders to plan intervention design \& locate suitable land

- Construction of wells, pipeline, and tanks for intervention, setup of field laboratory

- Pre-trial (baseline) household survey (T0), hygiene and safe water storage education

\section{Stage 2: Quantitative SW-CRT (Oct 2016 - Dec 2017)}

Delivery of piped untreated water (control condition) to tanks in all villages initially (T1)

- Sequential introduction of piped riverbank filtration-treated water (intervention condition) delivered to tanks in each village in random order at 12 week intervals (T2-T5)

- Health outcome data collected through five household surveys (T1-T5)

\begin{tabular}{|c|c|}
\hline \multicolumn{2}{|c|}{ Stage 3: Qualitative explanatory study (Jan 2018) } \\
\hline $\begin{array}{c}\text { Household focus groups/interviews } \\
(\mathrm{n}=21)\end{array}$ & $\begin{array}{c}\text { Surveyor focus groups } \\
(\mathrm{n}=4)\end{array}$ \\
\hline
\end{tabular}

FIGURE 1. Temporal summary of project stages and activities. SW-CRT = stepped-wedge cluster randomized trial; T0 = timepoint zero; $\mathrm{T} 1-\mathrm{T} 5=$ timepoints 1 to 5 . 
TABLE 1

Mapping of surveyor and household focus group questions about barriers and enablers to intervention uptake to the COM-B domains

\begin{tabular}{|c|c|c|}
\hline Household focus group & Surveyor focus group & Questions/statements \\
\hline \multicolumn{3}{|c|}{$\begin{array}{l}\text { Capability: the individual's psychological and physical capacity to engage in the activity concerned. It includes having appropriate knowledge and } \\
\text { skills }\end{array}$} \\
\hline $\boldsymbol{V}$ & & How do you choose which water to drink? \\
\hline $\boldsymbol{レ}$ & & How do you identify water of good quality? \\
\hline $\boldsymbol{レ}$ & & What do you do to ensure that your drinking water is safe? \\
\hline \multirow[t]{3}{*}{$\boldsymbol{\nu}$} & & How does the quality of your drinking water affect your health or the health of your children? \\
\hline & レ & What connection do householders see between water and health? \\
\hline & $\boldsymbol{\swarrow}$ & What do people in the village say about the quality of project tank water? \\
\hline \multicolumn{3}{|c|}{ Opportunity: the factors that lie outside the individual that make the behavior possible or prompt it } \\
\hline \multirow[t]{3}{*}{ レ } & & What (if anything) prevents you from collecting water from the project tank? \\
\hline & レ & What have you observed regarding the collection of project tank water? \\
\hline & $\boldsymbol{レ}$ & What do people in the village say about the location of the project tanks? \\
\hline \multicolumn{3}{|c|}{$\begin{array}{l}\text { Motivation: reflective and automatic mechanisms that activate or inhibit behavior. It includes habitual processes, emotional responding, and } \\
\text { analytical decision-making }\end{array}$} \\
\hline レ & & Which water do you prefer to drink and why? \\
\hline レ & & What feelings/thoughts do you associate with project tank water? Have these changed over time? \\
\hline $\boldsymbol{\swarrow}$ & & $\begin{array}{l}\text { What feelings/thoughts do you associate with water from the reverse osmosis facility? Have these } \\
\text { changed over time? }\end{array}$ \\
\hline \multirow[t]{4}{*}{$\boldsymbol{\nu}$} & & What do other people in your village think about project tank water? \\
\hline & レ & What have you heard householders say about the water in the project tanks? \\
\hline & レ & $\begin{array}{l}\text { How has the availability of a reverse osmosis water factory in the village impacted attitudes toward the } \\
\text { project tank water? }\end{array}$ \\
\hline & $\boldsymbol{\nu}$ & Do you think householders would be willing to pay for tank water? \\
\hline
\end{tabular}

only asked if they answered yes, we were also interested in exploring knowledge, understanding, and beliefs about illness and the impact the question structure may have had on health reporting. Focus groups were held at our field office or in meeting areas within study villages and were conducted in Kannada (the local language) by three bilingual (Kannada/ English) local researchers who were not directly involved in surveyor recruitment: a male moderator, female facilitator, and male scribe. Two female English-speaking researchers also attended and observed focus groups, documenting body language and interpersonal interactions. Following receipt of verbal consent to audiotape, focus groups were audiorecorded and field notes were taken by the scribe and English-speaking researchers. A light lunch was provided to participants as compensation for participation.
Household focus groups. Selection of households for focus groups was purposive, based on differing characteristics (village, household size, socioeconomic status, proximity to tanks, reported intervention uptake, and preferred drinking water source) determined from quantitative data. We conducted focus groups until we reached data saturation; this was determined when the analysis indicated that the focus groups were explaining the quantitative findings and the data from different focus groups were generating the same themes. Our approach to data collection from households was flexible. At each selected household, a written explanatory statement was provided and verbally explained to all household members and any additional study participants (family members or friends) who were present before commencement of the discussion. All those aged > 14 years who provided verbal

TABLE 2

Mapping of surveyor and household focus group questions about barriers and enablers to health reporting to the COM-B domains

\begin{tabular}{|c|c|c|}
\hline Household focus group & Surveyor focus group & Questions/statements \\
\hline \multicolumn{3}{|c|}{$\begin{array}{l}\text { Capability: the individual's psychological and physical capacity to engage in the activity concerned. It includes having appropriate knowledge and } \\
\text { skills }\end{array}$} \\
\hline レ & 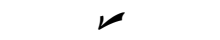 & Can you explain what the term illness/sickness means to you? \\
\hline \multirow[t]{4}{*}{ 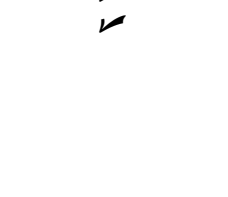 } & $\boldsymbol{\nu}$ & $\begin{array}{l}\text { Can you explain what the term diarrhea means to you? If you had diarrhea, would you say you were } \\
\text { sick/ill? }\end{array}$ \\
\hline & $\boldsymbol{\nu}$ & How do householders understand the term illness/sickness? What does it mean to them? \\
\hline & レ & Hov \\
\hline & レ & $\begin{array}{l}\text { How often did householders ask for further explanation of questions? Can you give an example? How did } \\
\text { you respond? }\end{array}$ \\
\hline \multicolumn{3}{|c|}{ Opportunity: the factors that lie outside the individual that make the behavior possible or prompt it } \\
\hline & $\boldsymbol{\nu}$ & Can you explain how you approached and askec \\
\hline & $\boldsymbol{\nu}$ & lestions? \\
\hline \multicolumn{3}{|c|}{$\begin{array}{l}\text { Motivation: reflective and automatic mechanisms that activate or inhibit behavior. It includes habitual processes, emotional responding, and } \\
\text { analytical decision-making }\end{array}$} \\
\hline & $\boldsymbol{\nu}$ & $\begin{array}{l}\text { Can you describe any differences you noticed in the way that people responded to survey questions? } \\
\text { Change over time? }\end{array}$ \\
\hline & レ & $\begin{array}{l}\text { Can you describe any differences you noticed in the willingness of householders to answer different types } \\
\text { of questions? Change over time? }\end{array}$ \\
\hline & レ & $\begin{array}{l}\text { What did you observe about householders' attitudes toward you, the survey or the project? Change over } \\
\text { time? }\end{array}$ \\
\hline
\end{tabular}


consent were invited to voice their opinions in a focus group discussion. Where only one household member was present, a semi-structured interview was conducted. Focus groups were conducted in participants' homes by the same research team as the surveyor focus groups. A question guide containing open-ended questions was developed and piloted with in-country research staff. Questions were designed to explore perceptions and beliefs about water and health, drivers of household water choices, and barriers and enablers to intervention uptake, and were mapped to the domains of the COM-B framework (Tables 1 and 2). Verbal consent to audiotape was sought before commencement. Participating households were provided with a water storage container at the end of the focus group as compensation for participation.

Data analysis. Focus group audio recordings were transcribed verbatim and translated from Kannada to English by an independent transcription service. Bilingual researchers who conducted focus groups checked transcripts against audio recordings to validate the data. Qualitative data were coded and analyzed in NVivo (Version 12, QSR International, Melbourne, Australia) by S. L. M., with inductive approaches using open, axial, and selective coding used to identify and explore emerging themes. Deductive approaches were also used to explore themes relating to intervention uptake and health reporting using the COM-B framework. ${ }^{28}$ A 20\% sample of transcripts was double coded by a second researcher (J. O.) to check for consistency in coding and theme development. A process of triangulation was applied at the interpretation stage of the analysis, whereby qualitative findings from household and surveyor groups and findings from quantitative surveys were considered to determine whether they were convergent, complementary, or contradictory. ${ }^{32}$ IIlustrative participant quotes were selected to support results and are presented with an additional text for clarification placed within square brackets as necessary.

Ethics. Ethics approval was obtained from the Monash University Human Research Ethics Committee in Australia (CF/15/522 2015000248) and the Energy and Resources Institute Ethics Committee in India, and the study was registered with the Australia New Zealand Clinical Trials Registry (ACTRN12616001286437).

\section{RESULTS}

Study participants. Baseline demographic characteristics of households enrolled in the stepped-wedge trial have previously been described. ${ }^{27} \mathrm{~A}$ summary of qualitative research activities and participants is provided in Table 3. All householders invited to participate in this qualitative study consented. We conducted 20 focus groups and one semistructured interview with householders, involving 50 participants from 28 households across the four villages. Selected households ranged in size from 1 to 13 persons and were evenly distributed across the four study villages. Between two and six participants were present at each focus group (median =2), and the average duration was 39 minutes (range: 28-50 minutes). The single semi-structured interview was conducted with a woman who lived alone. Hereafter, we collectively refer to focus groups and/or semi-structured interviews involving householders as "household focus groups." We conducted four focus groups involving 17 of the 24 surveyors; at least one member of each pair attended. The remaining seven surveyors declined to participate, citing other work commitments. Each focus group had 3-6 participants, and the average duration was 85 minutes (range: 61-104 minutes).

Intervention implementation and uptake. Implementation and uptake of our water intervention is described in detail in our quantitative article. ${ }^{27}$ Briefly, low intervention uptake rates were observed, potentially explained by the unexpected introduction of a competing water supply intervention (construction of reverse osmosis water facilities in study villages just before trial commencement) and issues with intervention fidelity (lack of continuous water supply to some tanks because of electricity shortages).

Barriers and enablers. In the following sections, we first describe the key themes emerging from the analysis of barriers and enablers to intervention uptake. We then describe the key themes emerging from the analysis of barriers and enablers to health reporting. In each section, results are reported thematically according to the domains of the COM-B framework ("capability," "opportunity," and "motivation"), with householder and surveyor responses combined under the relevant headings. Dominant themes within the data (those that were discussed in multiple focus groups and across different villages) are designated as "major themes"; less dominant themes (those only discussed within one village or in one or two focus groups) are designated as "minor themes."

Barriers and enablers to intervention uptake. Key themes emerging from the analysis of barriers and enablers to intervention uptake are summarized in Table 4, with each theme mapped to the relevant domain of the COM-B framework.

Capability. Distorted perceptions of water-related health effects (major theme). Lack of understanding of the health consequences of drinking contaminated water was a barrier to intervention uptake, and distorted perceptions of waterrelated health effects were common. Several householders expressed the belief that changing to a new drinking water source might make them or their children sick, and symptoms attributed to water source changes included cough, cold, throat pain, runny nose, joint pain, and headache, in addition to diarrhea, vomiting, stomach pain, and digestion issues.

Stomach ache, headache, whenever we change water it happens. (Female householder, village 4)

TABLE 3

Summary of research activities and participants

\begin{tabular}{|c|c|c|c|c|c|}
\hline \multirow[b]{2}{*}{ Activity type } & \multicolumn{4}{|c|}{ Participant(s) } & \multirow[b]{2}{*}{ Total duration } \\
\hline & Description & Number & Age (years), mean (range) & Male sex, $n(\%)$ & \\
\hline $\begin{array}{l}\text { Household focus group }(n=20) \text { or } \\
\text { semi-structured interview }(n=1)\end{array}$ & $\begin{array}{l}\text { Household members (aged } \geq 14 \text { years) } \\
\text { from } 28 \text { households }\end{array}$ & 50 & $40.7(15-75)$ & $22(44 \%)$ & 13 hours 17 minutes \\
\hline Surveyor focus group $(n=4)$ & Research assistants ("surveyors") & 17 & $25.5(19-49)$ & $16(94 \%)$ & 5 hours 43 minutes \\
\hline
\end{tabular}


TABLE 4

Mapping of barrier and enabler themes for uptake of intervention (project tank water) $^{*}$

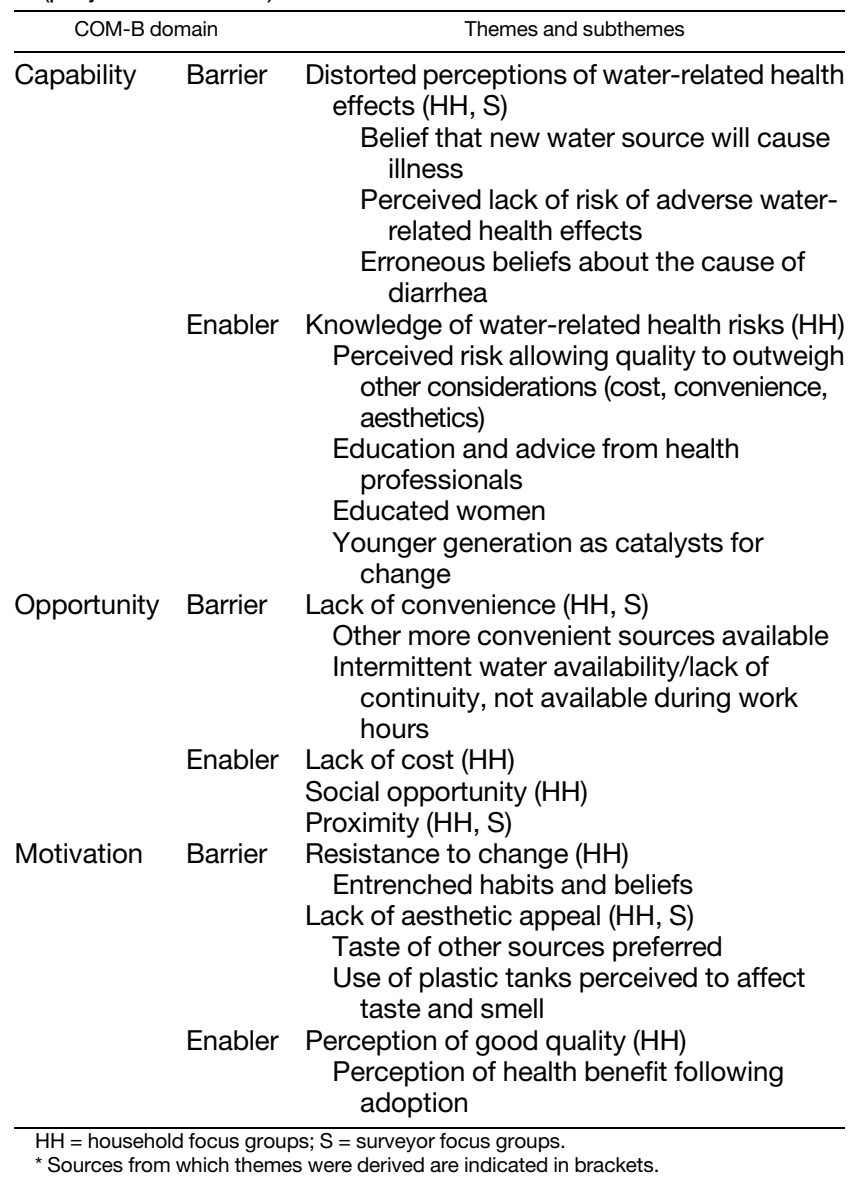

Some householders did not perceive any risk of adverse water-related health effects. For example, those who consumed untreated river water during agricultural work believed they were not at risk of illness as they were "used to it." Other householders believed adverse health consequences to be transient, and not concerning. As one man in village 4 explained, "When river water comes, we catch a little cold for a week and later we are fine."

Surveyors agreed that distorted perceptions of water-related health effects were common and that householders frequently attributed respiratory and systemic symptoms, as well as gastrointestinal symptoms, to changes in water source. Surveyors also reported that some householders did not perceive any health risks from untreated water sources, and others held erroneous beliefs about the cause of diarrhea (e.g., that it resulted from sun exposure or changes in body heat); these were felt to be barriers to uptake of safe water sources or practices.

"Today we drink filter water and some other day we drink river water-nothing happens to us." Some of them have this mentality. (Surveyor, village 2)

If their child has diarrhea, they say it comes because he roams in the sun... or because he eats sour food like tamarind. (Surveyor, village 1)
Knowledge of water-related health risks (major theme). Knowledge of water-related health risks was an enabler to uptake, especially if held by women, who were generally responsible for decision-making about household water source selection and treatment.

They [other household members] just eat and drink whatever we prepare. So whatever we decide they will agree Sir. So it is we who decide. (Female participant, village 1)

Women are of the opinion that we must only use clean water. (Male participant, village 2)

Householders with this knowledge were more likely to adopt a safe water source or effective water treatment practice, and more willing to go out of their way to get what they perceived to be the best-quality water source. For example, before the construction of reverse osmosis water treatment facilities in their own village, some householders had been traveling up to $15 \mathrm{~km}$ to collect reverse osmosis-treated water from a nearby village.

We used to go by two-wheeler [motorbike] even two years back to get [reverse osmosis-treated] water. ... we have stopped drinking river water as it is dirty. (Female householder, village 1)

Householders with knowledge of water-related health risks often described a hierarchy of safe water sources and treatment practices, with reverse osmosis-treated water and boiling typically at the top. Although some householders adopted safe sources and/or treatment practices yearround, others only adopted them at times of perceived increased risk (such as during the monsoon season), where quality outweighed other considerations such as cost, convenience, and aesthetic preferences. Other households only adopted safer sources when family members were visiting from out of town.

During the monsoon we compulsorily boil [river water] and during summer we sometimes boil. . . but during winter we consume as it is. (Male householder, village 3)

When children come for vacation, they can't drink river water, so we get filtered water. (Female householder, village 2)

Education about water-related health risks was generally imparted by a doctor or nurse. Attendance at a health facility for acute diarrhea often prompted a change in water practices based on doctor's recommendations. However, some householders reported that although they would follow the doctor's instructions during their illness, once recovered, they would return to their usual preferred practices:

Loose motion (diarrhea) is serious. . so we have to visit the doctor... the doctor tells us to boil and drink the water. (Male householder, village 3)

Only when we are sick we do so [boil water] as we have no other option. If women boil and give, then we drink. After [we recover] again we drink cold [untreated] water. (Male householder, village 4) 
Younger household members were often catalysts for change, providing education to older household members, encouraging them to adopt safe sources or treatment practices, and sometimes chastising them for going back to old habits:

For children and elders and all, if we boil and store and drink it is good. ... our son has taught us this. He scolds us whenever he sees [us drinking untreated water]. (Female householder, village 3)

My elder daughter has done M.Sc. in chemistry. She tells us to use and drink clean water... she has created awareness whenever she has come here. (Male householder, village 2)

Opportunity. Lack of convenience (major theme). The need to collect riverbank filtration-treated water from tanks was a barrier to uptake as other more convenient sources were available. Untreated river water was generally considered the most convenient water source, with all but one of the households interviewed having access to a standpipe within $10-50 \mathrm{~m}$ of their compound to which river water was supplied for about 2-3 hours morning and night (depending on electricity). Tanks were often perceived to be too far away to be of use, and some householders expressed a desire for direct supply to standpipes:

A few say it [tank water] is of no use as it is so far off. (Surveyor, village 1)

They would be happy if it was supplied directly to standpipes. (Surveyor, village 3)

The continuity of tank water supply was impacted by intermittent electricity availability in villages 1 and 2, with well yields sometimes insufficient to fill all project tanks. Both householders and surveyors described a sense of frustration among those located closest to the intermittently empty tanks:

They curse and ask [for the tank] to be filled. (Male participant, village 1)

We got a scolding for not supplying water. (Surveyor, village 1)

Another barrier to availability was accidental or deliberate damage to the tank infrastructure, or maintenance delays. Householders in village 3 reported that leakage from, or removal of, taps limited their ability to access water.

One tap leaks and it is repaired... another day children would have broken another. Some person should be committed to do this job and keep the water supply continuous. (Female householder, village 3)

In addition, tank water was not always accessible during work hours, particularly for agricultural workers:

Nobody stays at home continuously. Most of them work in the fields. Wherever they get water they drink. Be it river water or bore, they drink. (Surveyor, village 2)
Lack of cost (minor theme). The lack of cost associated with the tank water was reported by a number of householders as an enabler to intervention uptake. Adopters of tank water reported saving money on candle filters and/or fuel (used to boil water). However, the lack of cost did not appear to be a compelling reason to use tank water for those households who adopted reverse osmosis-treated water and were willing to pay for this.

Social opportunity (minor theme). Some householders were flexible and opportunistic and were willing to seek out and try new water sources, especially if recommended by social networks; this was an enabler to intervention uptake. Key triggers for trying new sources were positive word of mouth and observation of uptake by others.

When other people started going, only then we went. (Female householder, village 3)

The people say if we drink river water there might be a problem. This filter water is better. (Female householder, village 4)

A number of householders reported changing their main drinking water source over time as new sources or treatment options became available. As an example, one household reported changing from a candle filter to water from the reverse osmosis facility, to riverbank filtration-treated water within the space of about 2 years-these changes were prompted by positive peer opinions, and weighing the relative importance of cost, quality, and convenience. Of note, their adoption of riverbank filtration-treated water predated trial commencement and allocation of their village to the intervention arm, as they did not take it from a tank, but directly from a well.

The people who drilled [the RBF well] told us that the water is tasty and told us to try. So we started using this sweet water. After bringing it [home] once, we continued. (Male householder, village 2)

Proximity (minor theme). Proximity to tanks was described as an enabler to uptake, and households adopting tank water were typically located close to the tanks (within a 5-minute return trip). This was confirmed by surveyors.

Those who are near to the tank will take the water. (Surveyor, village 4)

Motivation. Resistance to change (minor theme). A resistance to changing water sources was encountered among some householders, most often among those of the older generation. Reasons for resistance included a reluctance to alter existing habits, a preference for certain water sources, and entrenched beliefs about the adverse health effects of changing sources. Householders who were "used to" their existing source often saw no compelling reason to change their behavior.

The children may catch cold if there is change in [drinking] water. If water from a single source is used, then health 
can be maintained. If there is a change, then it will be a problem. (Female householder, village 2)

We get digestion problems when we change water... but with river water, we will not have any symptoms. (Male householder, village 1)

Lack of aesthetic appeal (major theme). Taste was an important factor for many householders in determining whether to drink a particular water source. As a man from village 1 explained, "We taste and see, and if we find it is good, we shall start using it." Many householders reported a taste preference for river water, which was frequently described as "tasty." Reverse osmosis water was typically described as tasteless. Opinions on the taste of tank water were mixed; some householders disliked it, describing it as "salty" or "sour," others felt it was neutral or "tasteless," and a select few liked the taste, finding it to be "sweet," or in one case to taste "like Bisleri water," referring to a popular brand of mineral water in Southern India. Surveyors reported hearing that the water tasted salty or sour, and felt that taste was a barrier to uptake. Because of the taste, some householders would only resort to drinking tank water when other more tasty water sources were not available. As one woman in village 3 explained: "It was a little hard, tasteless, not very sweet, but the river was dry and so we were drinking that."

The use of plastic storage tanks was perceived by some householders to affect the taste and smell of water. These views were reinforced by surveyors. Some householders reported a preference to store water in steel containers or mud pots (rather than plastic) to help keep it cool and reduce any taste disturbance; this practice was frequently observed at household focus group visits and in photographs taken of household stored water containers at survey visits.

In summer plastic stinks. .. it gets heated up... so we don't like drinking from it. . . It doesn't quench the thirst... We don't store drinking water in plastic containers. (Female householder, village 3)

Perception of good quality (minor theme). Householders were more likely to adopt a new water source if they perceived it to be of good quality. For some householders, quality was judged on the taste and appearance; as a woman in village 4 explained, "It should be clean to look at and tasty also." For others, water was of good quality if it made them "feel good" or was not associated with adverse health effects; as one man in village 4 reported, "After drinking we had no health problems, so we started using that." One household in village 1 reported that reverse osmosis water was of good quality because it had been produced using a high-tech process: "It is cleaned by machine," and the household drank this water even though the taste was felt to be a "little sour."

Barriers and enablers to health reporting. Key themes emerging from the analysis of barriers and enablers to health reporting are summarized in Table 5, with each theme mapped to the relevant domain of the COM-B framework.

Capability. Variability in interpretation of what constitutes illness (minor theme). Surveyors believed that householders generally perceived illness (sickness) as being "not well." Although diarrhea was generally considered to constitute "illness," some surveyors believed that relatively mild diarrheal
TABLE 5

Mapping of barrier and enabler themes for health reporting*

\begin{tabular}{|c|c|c|}
\hline \multicolumn{2}{|c|}{ COM-B domain } & Themes and subthemes \\
\hline Capability & Barrier & $\begin{array}{l}\text { Variability in interpretation of what } \\
\text { constitutes illness }(\mathrm{HH}, \mathrm{S}) \\
\text { Question interpretation and language (S) }\end{array}$ \\
\hline Opportunity & Barrier & $\begin{array}{l}\text { Short cuts to expedite survey completion (S) } \\
\text { Assumptions based on insider knowledge }\end{array}$ \\
\hline & Enabler & $\begin{array}{l}\text { Surveyor initiatives to encourage reporting (S) } \\
\text { Persistent attempts to elicit reports of } \\
\text { illness }\end{array}$ \\
\hline Motivation & Barrier & $\begin{array}{l}\text { Disengagement from the study }(\mathrm{S}) \\
\text { Weariness in answering repetitive } \\
\quad \text { questions } \\
\text { Perceived inaction by study team on health } \\
\text { data already provided (S) } \\
\text { Wariness in disclosing sensitive information } \\
(\mathrm{HH}, \mathrm{S})\end{array}$ \\
\hline & Enabler & Reporting illness for the common good $(\mathrm{HH})$ \\
\hline
\end{tabular}

$\mathrm{HH}=$ household focus groups; $\mathrm{S}$ = surveyor focus groups

* Sources from which themes were derived are indicated in brackets.

symptoms were often considered trivial and might not be reported. When surveyors asked about illness, most householders responded with reports of symptoms such as fever, cough, cold, runny nose, diarrhea (loose motion), and vomiting. Similarly, householders in focus groups reported that symptoms such as cough, cold, fever, stomach ache, diarrhea, vomiting, or problems with digestion equated to illness. However, some householders had a very different interpretation, feeling that the term illness referred to physical ailments and injuries. As one surveyor from village 2 explained, "If we tried questioning them, they would not give us a report about vomiting or loose motions; they just told us about breaking limbs 6 months ago and so on."

Question interpretation and language (minor theme). Surveyors reported that householders sometimes had difficulty in understanding questions, and they would often need to repeat questions or reframe them in local (colloquial) language. When surveyors felt that understanding was limited, they would provide a list of options or elaborate on the answers they were looking for, but in doing so, they often preempted or misguided householders' responses or reframed the question in such a way that the original intent was lost.

Some of the questions they can answer, but others we have to explain and give options-only then can they answer. (Surveyor, village 4)

This question [about illness] they understood as physical damage... so we had to explain... we had to ask was there any illness due to water. (Surveyor, village 2)

Opportunity. Shortcuts to expedite survey completion (minor theme). Surveyor pairs were assigned households to survey at each timepoint and asked to conduct their surveys within a 6-week period. As many householders engaged in agricultural work were not home during the day, and surveyors were frequently engaged in other forms of employment, surveyors typically conducted surveys at times or on days when more people were at home. The need to complete surveys in a timely fashion appeared to impact on the fidelity of survey implementation, as some surveyors took shortcuts to expedite survey completion. For example, surveyors from village 4 reported that if they came across a group of householders 
sitting together in a public place, they would conduct surveys for multiple households concurrently.

Surveyors were community members and had existing social ties with some householders. This gave them insider knowledge about habits and practices but impacted on their objectivity. Surveyors admitted that when conducting surveys with community members they knew, they would preempt responses to some questions to expedite survey completion-this was sometimes encouraged by householders. Furthermore, if householders provided a response that was perceived by surveyors to be untruthful, they would sometimes enter what they believed to be the correct response:

Sometimes they [householders] say "you know it-you may write it yourself." (Surveyor, village 1)

Sometimes about smoking they don't tell. . you tick that because you know. (Surveyor, village 4)

Surveyor initiatives to encourage reporting (minor theme). The higher rates of symptom reporting seen in village 3 relative to other villages may be explained in part by the village 3 surveyors' approach to the survey. Although they received the same training as the other surveyors, they met regularly on their own initiative to discuss how best to engage and maintain interest. Following the baseline survey (and unbeknownst to the primary research team), they brainstormed an introductory statement for each subsequent survey with invented objectives (such as to further investigate "water quality," "smoke exposure," or "household expenditure on water") to maintain interest. Although other surveyor teams took negative responses to questions about illness at face value, surveyors from village 3 , convinced that there was more illness going on than was being reported, decided to be persistent and go "off-script" to elicit reports of illness by saying things like:

No problem, just tell us, it won't cost you anything to answer! (Surveyor, village 3)

Motivation. Disengagement from the study (major theme). Surveyors reported that engagement and interest in the study waned over time. This was partially attributed to a lack of perceived direct benefit from the study, particularly among households located at greater distances from project tanks, or in villages where tanks were intermittently empty. Some surveyors felt that those who saw a benefit from the intervention were more willing to answer questions and more likely to answer truthfully:

Initially they answered the survey. . later they were not interested. They asked us, "what is the benefit?" (Surveyor, village 2)

Our water didn't reach them so they asked "when your water is not reaching us why do you ask questions?" (Surveyor, village 1)

Anyone who is close to a tank answered happily as they benefited. . . the ones who are far answered a little sadly. (Surveyor, village 2)

Surveyors observed that participants tired of answering the same questions repeatedly and became less engaged as the survey questions wore on. As questions relating to health were asked toward the end of the survey, and were repeated at each survey, this may have influenced the likelihood of answering health questions.

[During the survey] their enthusiasm decreases. . . they ask us "how many more questions are left" [to answer]. (Surveyor, village 1)

Surveyors also felt that participants were less likely to engage in the surveys and answer all the questions if they perceived the survey to be taking them away from other priorities:

If they are free, they tell but if they are busy, they get irritated. (Surveyor, village 4)

Perceived inaction by study team on health data already provided (major theme). Surveyors reported that although participants were generally willing to report their health symptoms in the initial pretrial (TO) survey, many did not perceive a benefit in continuing to report health symptoms because of a perception of inaction by the study team in response to previous reports:

There were a few complaints. When we came last time they gave us the report [of symptoms of illness] and what is the use? No use na? What did we do in taking their reports? Nothing... After the first survey they expected some solution. (Surveyor, village 2)

They said 'you come and ask about our sickness and go but what is the use [of reporting] if there is no response from your side'? (Surveyor, village 3)

Some surveyors believed that those who had already received treatment for their illness might not bother to report symptoms because of a sense of futility:

They went to hospital right, they might have felt that even if they tell us [their symptoms] there is no use. (Surveyor, village 3)

Wariness in disclosing sensitive information (minor theme). Surveyors perceived some householders to be wary about disclosing health problems and reported being met with resistance and suspicion in some households when they asked about health. They believed that some householders were worried that they might be asked to disclose a history of sensitive medical issues such as HIV or tuberculosis, and this made them less willing or unwilling to answer questions about health.

Sometimes they fear what we would do in taking these data. So [when we ask if anyone has suffered illness] they say no. (Surveyor, V4)

During household focus groups, one male householder from village 4 expressed reluctance in disclosing health information, particularly when relating to the presence of blood in bodily secretions (e.g., in stool or sputum).

Reporting illness for the common good (minor theme). During focus groups, most householders appeared to be happy to answer questions about their health. Some householders 
perceived that sharing information about their health had the potential to benefit them or their community. For example, one woman from village 1 described a sense of social responsibility in reporting symptoms: "If they are asking about health, then why should we hide [our symptoms]? If we hide, then it will be a problem for all of us."

\section{DISCUSSION}

This theory-informed qualitative explanatory study identified important barriers to intervention uptake and health reporting in a water intervention trial in rural India. The mixedmethods approach to data analysis (through triangulation of quantitative and qualitative data) provided new insights into quantitative trial findings. Qualitative findings also provide broader insight into obstacles and facilitators to implementing and evaluating community water interventions, and ways in which these might be overcome or exploited to improve intervention uptake and achieve better health reporting in future studies.

Barriers to intervention uptake were multifactorial and included inability to perceive a health benefit in changing existing water practices, lack of convenience, and absence of motivation due to entrenched habits and beliefs, fear of the new and limited aesthetic appeal. Enablers to intervention uptake included knowledge of water-related health risks, proximity to tanks, social opportunity, and motivation through perception of good quality. Despite the availability of improved water sources in the form of both riverbank filtration-treated water and new reverse osmosis water treatment facilities, many householders expressed an ongoing preference for river water. In addition, households frequently reported contemporaneous use of more than one drinking water source, or switches between water sources according to season, preference, convenience, and perceptions of quality. Health gains from an improved water source are likely to be limited where consumption of lower quality sources continues. To maximize health gains, it is therefore necessary to work toward solutions that are sufficiently acceptable to allow exclusive use and/or that minimize the likelihood of nonadherence by reducing the burden on the user to change behavior. ${ }^{10,33,34}$ Observed inconsistencies in health beliefs relating to water were consistent with previous studies in rural India, showing that beliefs about the cause of diarrhea vary widely, and consumption of unsafe water is often linked to other unrelated symptoms such as cough and cold. ${ }^{35,36}$ Although education about the negative health consequences of contaminated water consumption by health professionals was often a catalyst for the adoption of safe treatment practices or use of a safe source, such practices often proved difficult to sustain. These observations reinforce that provision of safe water in the absence of strategies to promote behavior change is insufficient to alter entrenched habits and beliefs.

Perceptions of water-related health effects were an important barrier to intervention uptake identified in this study. We labeled perceptions (e.g., that consumption of untreated river water would not result in deleterious health effects) as "distorted" if they did not conform to the current scientific understanding about the risk of enteric pathogen transmission through contaminated water. Nevertheless, findings from recent high-quality water intervention trials in LMICs have shown mixed effects on child diarrhea despite high implementation fidelity and intervention uptake, which has increased uncertainty regarding the efficacy of water interventions in preventing diarrhea. ${ }^{17-19}$ These findings of themselves do not refute the biological plausibility that improved water quality can reduce diarrhea, but they do suggest that low-cost water interventions currently available to rural dwellers in LMICs might not sufficiently reduce the high levels of fecal contamination in household environments to result in an impact on health. ${ }^{22,37}$ Such findings, if not appropriately portrayed to at-risk populations, could potentially lead to mixed messages about the health benefits of water treatment, which could further impact the uptake of water interventions.

We approached our trial design from a technological and regulatory mindset, rather than a behavior change mindset. Hydrogeologic characteristics for riverbank filtration system installation and preexisting relationships with Karnataka municipal authorities established during a previous World Bank study ${ }^{38}$ were primary drivers for selection of our village study sites. Intervention planning discussions were held with government and local village leaders, but not with householders themselves. In retrospect, had we engaged with householders at the outset to identify capabilities and motivators, some relevant modifications to intervention delivery and strategies to maximize uptake could potentially have been implemented, such as addressing palatability and geographical tank coverage. Some factors remained outside our control, including introduction of a competing water supply (reverse osmosis facilities) by the state government, but changes in policy are always a risk with long trials where the process of planning to completion often takes 3-5 years. Although we delivered safe water storage and hygiene education, we did not attempt to influence uptake or adoption of our water intervention, in part because we did not want to alert participants of the switch from untreated river water to riverbank filtration-treated water in the stepped-wedge design. The absence of promotional and participatory activities relating to intervention delivery meant that many households were not appropriately motivated to use the systems introduced. Greater vigilance in implementation fidelity monitoring and rapid response mechanisms throughout the trial, such as those demonstrated in trials like WASH Benefits, ${ }^{39}$ may have improved uptake of our intervention.

Barriers to health reporting included reporting fatigue, perceived inaction on health data already provided, and inconsistencies in survey administration including conducting surveys in public places. Enablers included a sense of social responsibility in reporting health conditions to benefit family and community. Higher levels of education generally correlated with a better understanding of health states, respect for medical advice, and the importance of consultation for medical conditions. In village 3 , surveyor initiatives to maintain engagement and encourage reporting appear to have helped elicit ongoing illness reports. Had an exploratory qualitative study been performed before our trial, we might have been better able to design and implement our health survey to maximize sustained health reporting (e.g., by providing nominal value culturally appropriate incentives to participating households), reduce variability in concepts of illness, and potentially minimize deliberate (to terminate the survey quickly) or unintentional (due to recall bias) underreporting of symptoms. The use of a 7-day recall period may also have impacted on health reporting, although differing opinions exist 
as to the optimal recall period for subjective illness reporting methods; some researchers discourage the use of recall periods longer than 2-3 days to avoid underestimating incidence and selectively capturing more severe episodes, ${ }^{40,41}$ and others suggest that a 7-day recall period is optimal for statistical efficiency. ${ }^{42,43}$ In contrast to other studies, where a household "index case" is chosen for monitoring, ${ }^{17}$ we asked a single respondent to report illness episodes for all household members which may also have impacted on reporting rates.

Locally recruited surveyors had excellent knowledge of local dialects and village settings and employing local community members was designed to build capacity and increase acceptability. However, externally recruited surveyors may have been more objective and consistent in survey administration, reducing both observer and responder biases. Our findings highlight the need for thorough and ongoing surveyor training, including cultural and language education, and systems to measure and monitor surveyor performance. Relevant markers of surveyor performance might include monitoring survey duration (easily obtainable with electronic survey delivery methods), GPS location (to determine where surveys are being conducted), survey completeness, and comparative rates of health reports elicited.

There are important limitations to the conclusions that can be drawn from this research. First, we studied a communitybased water intervention delivered to four rural villages in a single region of India, and it is possible that barriers and enablers to intervention uptake and health reporting identified here might not be relevant to other settings. Second, although individuals were encouraged to express alternate viewpoints through probing questions, there is potential for focus groups to generate confirmation bias and "socially desirable replies," although this was overcome to an extent through triangulation of different data sources. Third, the use of purposive sampling when selecting households for focus groups may have led to selection bias, although we did our best to ensure that households with differing demographics and preferred water sources were represented. Finally, qualitative data were analyzed in English based on transcripts translated from Kannada. Although we used a professional transcription and translation service, and bilingual researchers involved in conducting focus groups checked transcripts against audio recordings for validation, it is possible that some nuances of meaning were lost in translation.

Our use of the COM-B model and a sequential explanatory mixed-methods study approach allowed us to better understand trial results, identify implementation and evaluation barriers, and consider ways they might be overcome in future studies. Our findings highlight the vital need to collect qualitative exploratory data before trial commencement to fully understand local contexts and inform the design and delivery of the intervention and strategies to maximize and maintain uptake, adherence, and participation. Our findings also support recent calls to improve reporting of intervention fidelity and outcome measurement, and integrate process evaluations into trials of complex interventions. ${ }^{22,23,44-46}$ Application of implementating science frameworks to these trials, as used in other public health disciplines, could further allow identification of key barriers and enablers to intervention delivery and utilization, and support understanding of how to replicate and scale successful interventions. ${ }^{23,46-48}$ Future trials should aim for high coverage and exclusive use of intervention water supplies, using structured, real-time fidelity monitoring and response systems, to detect and rapidly correct barriers to sustained intervention uptake. ${ }^{39,46}$ Surveyor training and monitoring of survey implementation are also important, combined with a suitable timely response system to address lapses as they occur.

Received June 28, 2019. Accepted for publication December 15, 2019.

Published online January 13, 2020.

Note: Supplemental Appendix appears at www.ajtmh.org.

Acknowledgments: We thank all the staff at the Energy and Resources Institute in India, particularly Kavita Patil and Fraddry D'Souza for coordinating personnel, venue and travel arrangements, and transcription and translation services; and Veerabaswant Reddy for moderating focus groups. We also thank our collaborators Thomas B. Boving (University of Rhode Island, USA) and Andrew B. Forbes (Monash University, Australia) for their participation and feedback in workshop discussions and teleconferences. Finally, we thank the surveyors and householders who took part in this study for their time, patience, and candor.

Financial support: This research was supported by an Australian National Health and Medical Research Council (NHMRC) project grant (1083408). K. L. is supported by an NHMRC Research Fellowship (1155005), A. C. C. by an NHMRC Career Development Fellowship (1068732), and S. L. M. by a NHMRC Postgraduate Scholarship (1115196).

Authors' addresses: Sarah L. McGuinness, Joanne O'Toole, Darshini Ayton, Allen C. Cheng, and Karin Leder, School of Public Health and Preventive Medicine, Monash University, Melbourne, Australia, E-mails: sarah.mcguinness@monash.edu, joanne.otoole@monash.edu, darshini.ayton@monash.edu, allen.cheng@monash.edu, and karin.leder@ monash.edu. Asha Giriyan, Chetan A. Gaonkar, and Ramkrishna Vhaval, The Energy and Resources Institute (TERI), Santa Cruz, India, E-mails: ashag@teri.res.in, chetannio@gmail.com, and r.vhaval@teri.res.in.

\section{REFERENCES}

1. Howitt $P$ et al., 2012. Technologies for global health. Lancet 380 : 507-535.

2. Landrigan PJ et al., 2018. The Lancet Commission on pollution and health. Lancet 391: 462-512.

3. Bain R et al., 2014. Global assessment of exposure to faecal contamination through drinking water based on a systematic review. Trop Med Int Health 19: 917-927.

4. Pruss-Ustun A et al., 2014. Burden of disease from inadequate water, sanitation and hygiene in low- and middle-income settings: a retrospective analysis of data from 145 countries. Trop Med Int Health 19: 894-905.

5. GBD Diarrhoeal Diseases Collaborators, 2017. Estimates of global, regional, and national morbidity, mortality, and aetiologies of diarrhoeal diseases: a systematic analysis for the Global Burden of Disease Study 2015. Lancet Infect Dis 17: 909-948.

6. Bain R, Cronk R, Wright J, Yang H, Slaymaker T, Bartram J, 2014. Fecal contamination of drinking-water in low- and middleincome countries: a systematic review and meta-analysis. PLoS Med 11: e1001644.

7. Wolf $\mathrm{J}$ et al., 2018. Impact of drinking water, sanitation and handwashing with soap on childhood diarrhoeal disease: updated meta-analysis and meta-regression. Trop Med Int Health 23: 508-525.

8. WHO/UNICEF Joint Monitoring Programme for Water Supply Sanitation and Hygiene, 2017. Progress on Drinking Water, Sanitation and Hygiene: 2017 Update and SDG Baselines. Geneva, Switzerland: World Health Organization and the United Nations Children's Fund.

9. Hutton G, Chase C, 2016. The knowledge base for achieving the sustainable development goal targets on water supply, sanitation and hygiene. Int J Environ Res Public Health 13: E536. 
10. Brown J, Clasen T, 2012. High adherence is necessary to realize health gains from water quality interventions. PLoS One 7: e36735.

11. Hunter PR, 2009. Household water treatment in developing countries: comparing different intervention types using metaregression. Environ Sci Technol 43: 8991-8997.

12. Boisson S, Kiyombo M, Sthreshley L, Tumba S, Makambo J, Clasen T, 2010. Field assessment of a novel household-based water filtration device: a randomised, placebo-controlled trial in the Democratic Republic of Congo. PLoS One 5: e12613.

13. Luby SP, Mendoza C, Keswick BH, Chiller TM, Hoekstra RM, 2008. Difficulties in bringing point-of-use water treatment to scale in rural Guatemala. Am J Trop Med Hyg 78: 382-387.

14. Arnold B, Arana B, MausezahI D, Hubbard A, Colford JM Jr., 2009. Evaluation of a pre-existing, 3-year household water treatment and handwashing intervention in rural Guatemala. Int J Epidemiol 38: 1651-1661.

15. Martin NA, Hulland KRS, Dreibelbis R, Sultana F, Winch PJ, 2018. Sustained adoption of water, sanitation and hygiene interventions: systematic review. Trop Med Int Health 23: 122-135.

16. Clasen TF, Alexander KT, Sinclair D, Boisson S, Peletz R, Chang $\mathrm{HH}$, Majorin F, Cairncross S, 2015. Interventions to improve water quality for preventing diarrhoea. Cochrane Database Syst Rev 10: CD004794.

17. Luby SP et al., 2018. Effects of water quality, sanitation, handwashing, and nutritional interventions on diarrhoea and child growth in rural Bangladesh: a cluster randomised controlled trial. Lancet Glob Health 6: e302-e315.

18. Null C et al., 2018. Effects of water quality, sanitation, handwashing, and nutritional interventions on diarrhoea and child growth in rural Kenya: a cluster-randomised controlled trial. Lancet Glob Health 6: e316-e329.

19. Humphrey $\mathrm{JH}$ et al., 2019. Independent and combined effects of improved water, sanitation, and hygiene, and improved complementary feeding, on child stunting and anaemia in rural Zimbabwe: a cluster-randomised trial. Lancet Glob Health 7: e132-e147.

20. Boisson S, Stevenson M, Shapiro L, Kumar V, Singh LP, Ward D, Clasen T, 2013. Effect of household-based drinking water chlorination on diarrhoea among children under five in Orissa, India: a double-blind randomised placebo-controlled trial. PLoS Med 10: e1001497.

21. Clasen T et al., 2014. Effectiveness of a rural sanitation programme on diarrhoea, soil-transmitted helminth infection, and child malnutrition in Odisha, India: a cluster-randomised trial. Lancet Glob Health 2: e645-e653.

22. Pickering AJ et al., 2019. The WASH Benefits and SHINE trials: interpretation of WASH intervention effects on linear growth and diarrhoea. Lancet Glob Health 7: e1139-e1146.

23. Mbuya MN, Jones $A D$, Ntozini R, Humphrey JH, Moulton LH, Stoltzfus RJ, Maluccio JA; Sanitation Hygiene Infant Nutrition Efficacy (SHINE) Trial Team, 2015. Theory-driven process evaluation of the SHINE trial using a program impact pathway approach. Clin Infect Dis 61 (Suppl 7): S752-S758.

24. Dreibelbis R, Winch PJ, Leontsini E, Hulland KR, Ram PK, Unicomb L, Luby SP, 2013. The integrated behavioural model for water, sanitation, and hygiene: a systematic review of behavioural models and a framework for designing and evaluating behaviour change interventions in infrastructurerestricted settings. BMC Public Health 13: 1015.

25. Clasen T, Smith KR, 2019. Let the "A" in WASH stand for air: integrating research and interventions to improve household air pollution (HAP) and water, sanitation and hygiene (WaSH) in low-income settings. Environ Health Perspect 127: 25001.

26. Thompson LM, Diaz-Artiga A, Weinstein JR, Handley MA, 2018. Designing a behavioral intervention using the COM-B model and the theoretical domains framework to promote gas stove use in rural Guatemala: a formative research study. BMC Public Health 18: 253.

27. McGuinness SL et al., 2020. A stepped wedge cluster-randomized trial assessing the impact of a riverbank filtration intervention to improve access to safe water on health in rural India. Am J Trop Med Hyg 102: 497-506.
28. Michie S, van Stralen MM, West R, 2011. The behaviour change wheel: a new method for characterising and designing behaviour change interventions. Implement Sci 6: 42.

29. Fetters MD, Curry LA, Creswell JW, 2013. Achieving integration in mixed methods designs-principles and practices. Health Serv Res 48: 2134-2156.

30. McGuinness SL, O'Toole JE, Boving TB, Forbes AB, Sinclair M, Gautam SK, Leder K, 2017. Protocol for a cluster randomised stepped wedge trial assessing the impact of a community-level hygiene intervention and a water intervention using riverbank filtration technology on diarrhoeal prevalence in India. BMJ Open 7: e015036.

31. Tong A, Sainsbury P, Craig J, 2007. Consolidated criteria for reporting qualitative research (COREQ): a 32-item checklist for interviews and focus groups. Int $J$ Qual Health Care 19: 349-357.

32. O'Cathain A, Murphy E, Nicholl J, 2010. Three techniques for integrating data in mixed methods studies. BMJ 341: c4587.

33. Humphrey $\mathrm{JH}, 2019$. Reducing the user burden in WASH interventions for low-income countries. Lancet Glob Health 7: e1158-e1159.

34. Pickering AJ, CriderY, Sultana S, Swarthout J, Goddard FG, Anjerul Islam S, Sen S, Ayyagari R, Luby SP, 2019. Effect of in-line drinking water chlorination at the point of collection on child diarrhoea in urban Bangladesh: a double-blind, cluster-randomised controlled trial. Lancet Glob Health 7: e1247-e1256.

35. Banda $\mathrm{K}$ et al., 2007. Water handling, sanitation and defecation practices in rural southern India: a knowledge, attitudes and practices study. Trans $R$ Soc Trop Med Hyg 101: 1124-1130.

36. Francis MR, Nagarajan G, Sarkar R, Mohan VR, Kang G, Balraj V, 2015. Perception of drinking water safety and factors influencing acceptance and sustainability of a water quality intervention in rural southern India. BMC Public Health 15: 731.

37. Cumming $O$ et al., 2019. The implications of three major new trials for the effect of water, sanitation and hygiene on childhood diarrhea and stunting: a consensus statement. BMC Med 17: 173.

38. Boving TB, Choudri BS, Cady P, Cording A, Patil K, Reddy V, 2014. Hydraulic and hydrogeochemical characteristics of a riverbank filtration site in rural India. Water Environ Res 86: 636-648.

39. Rahman M et al., 2018. WASH Benefits Bangladesh trial: system for monitoring coverage and quality in an efficacy trial. Trials 19: 360.

40. Feikin DR, Audi A, Olack B, Bigogo GM, Polyak C, Burke H, Williamson J, Breiman RF, 2010. Evaluation of the optimal recall period for disease symptoms in home-based morbidity surveillance in rural and urban Kenya. Int J Epidemio/ 39: 450-458.

41. Zafar SN, Luby SP, Mendoza C, 2010. Recall errors in a weekly survey of diarrhoea in Guatemala: determining the optimal length of recall. Epidemiol Infect 138: 264-269.

42. Arnold BF, Galiani S, Ram PK, Hubbard AE, Briceno B, Gertler PJ, Colford JM Jr., 2013. Optimal recall period for caregiverreported illness in risk factor and intervention studies: a multicountry study. Am J Epidemiol 177: 361-370.

43. Schmidt WP, Arnold BF, Boisson S, Genser B, Luby SP, Barreto ML, Clasen T, Cairncross S, 2011. Epidemiological methods in diarrhoea studies - an update. Int J Epidemiol 40: 1678-1692.

44. Moore GF et al., 2015. Process evaluation of complex interventions: medical research council guidance. BMJ 350: h1258.

45. Ridde V, 2016. Need for more and better implementation science in global health. BMJ Glob Health 1: e000115.

46. Vaz Nery S et al., 2019. The role of water, sanitation and hygiene interventions in reducing soil-transmitted helminths: interpreting the evidence and identifying next steps. Parasit Vectors 12: 273.

47. Peters DH, Adam T, Alonge O, Agyepong IA, Tran N, 2013. Implementation research: what it is and how to do it. BMJ 347 : f6753.

48. Oakley A, Strange V, Bonell C, Allen E, Stephenson J, Team RS, 2006. Process evaluation in randomised controlled trials of complex interventions. BMJ 332: 413-416. 